\title{
Néolithisation et néolithique en cyrénaïque (Libye)
}

\section{Elodie de Faucamberge}

\section{OpenEdition}

\section{Journals}

Édition électronique

URL : https://journals.openedition.org/encyclopedieberbere/2720

DOI : 10.4000/encyclopedieberbere. 2720

ISSN : 2262-7197

\section{Éditeur}

Peeters Publishers

\section{Édition imprimée}

Date de publication : 5 octobre 2012

Pagination : 5474-5481

ISBN : 978-90-429-2718-6

ISSN : $1015-7344$

\section{Référence électronique}

Elodie de Faucamberge, « Néolithisation et néolithique en cyrénaïque (Libye) », Encyclopédie berbère [En ligne], 34 | 2012, document N43, mis en ligne le 15 décembre 2020, consulté le 17 février 2022 URL : http://journals.openedition.org/encyclopedieberbere/2720; DOI : https://doi.org/10.4000/ encyclopedieberbere. 2720

Ce document a été généré automatiquement le 17 février 2022.

(c) Tous droits réservés 


\title{
Néolithisation et néolithique en cyrénaïque (Libye)
}

\author{
Elodie de Faucamberge
}

1 La Libye a connu un bel élan de recherches au cours du siècle dernier grâce à l'engouement des chercheurs et des amateurs pour l'art rupestre. Les investigations archéologiques ont commencé dans les années 1950, presque simultanément dans les trois grandes régions libyennes : la Tripolitaine, la Cyrénaïque et le Fezzan. Cependant seule cette dernière région et plus particulièrement le massif de l'Acacus a bénéficié d'importantes recherches préhistoriques jusqu'à la fin $\mathrm{du} \mathrm{XX}^{\mathrm{e}}$ siècle; les régions septentrionales de la Libye ont, quant à elles, été longtemps négligées. En Cyrénaïque, de récentes études menées parallèlement sur deux sites ont livré des informations nouvelles sur la période néolithique, notamment sur les débuts de la production de nourriture et de la production de céramique dans cette région.

2 La Cyrénaïque, au nord-est de la Libye, est principalement représentée par le massif du Djebel Akhdar qui borde la côte méditerranéenne sur toute sa partie septentrionale. La «Montagne Verte » culmine à un peu moins de $900 \mathrm{~m}$ d'altitude, mais ses sommets les plus élevés se trouvent tous près du littoral, arrêtant ainsi les nuages chargés de pluie venant de la Méditerranée. Cette configuration topographique permet au Djebel d'être recouvert d'une végétation typiquement méditerranéenne. Le massif possède un important réseau hydrographique de surface, aujourd'hui asséché, et plusieurs sources d'eau, grâce à l'imperméabilité des marnes et des argiles présentes dans les formations calcaires (Kleinsmiede \& Van Den Berg 1968). Les abris et grottes façonnés par l'érosion sont nombreux ; quelques-uns ont été fouillés au $\mathrm{XX}^{\mathrm{e}}$ siècle mais seule la grotte d'Haua Fteah contenait des dépôts holocènes en place. Malgré l'exceptionnelle découverte de ce site dans les années 1950, les recherches préhistoriques se sont interrompues dans la région pendant un demi-siècle. 


\section{Haua Fteah}

Le site d'Haua Fteah se trouve tout au nord de la Cyrénaïque, dans le Djebel Akhdar, à quelques kilomètres à l'est de la ville de Susa. La grotte a été découverte par C. B. M. McBurney et C. T. Houlder en 1948; elle fut étudiée en 1951, 1952 et 1955 (McBurney 1967). D'une superficie au sol de $100 \mathrm{~m}^{2}$, la fouille est descendue jusqu'à 13 mètres de profondeur, ce qui en fait l'une des plus longues stratigraphies d'Afrique du Nord. Trente-cinq couches ont été identifiées et regroupées en six étages préhistoriques: quatre pour le Pléistocène et deux pour l'Holocène. McBurney note un niveau préAurignacien libyen, un niveau Levalloiso-moustérien, un niveau Dabbéen, un niveau Eastern Oranian, un niveau Libyco-capsien et un niveau Néolithique dit de tradition capsienne à variante libyenne. Enfin le sommet du dépôt est représenté par une phase d'occupation historique. La couche VIII contenait des tessons de céramique et des os d'animaux domestiques marquant ainsi le début du Néolithique qui se termine avec la couche VI. Les quatre datations obtenues sur charbons par McBurney à l'époque pour le Néolithique sont les suivantes (fig. 1) : $6800 \pm 350$ BP et $6370 \pm 103$ BP (couche VIII), $5800 \pm 108$ et $4860 \pm 97 \mathrm{BP}$ (couche VI).

4 Au Néolithique, le premier changement dans la composition du matériel lithique par rapport aux industries précédentes est marqué par le déclin des lames à bord abattu qui ne représentent plus qu'un tiers de l'industrie. McBurney note une forte augmentation des grattoirs (environ $30 \%$ pour la couche VIII). Les burins augmentent aussi : c'est la troisième classe la mieux représentée. Le reste de l'industrie comprend des racloirs, de grandes et moyennes lames retouchées (dont des lames à coches), quelques perçoirs et des lames ou lamelles à double bord abattu. Les microburins et microlithes géométriques* sont très rares. Cinq couteaux (des éclats retouchés latéralement par pression), une vingtaine de pointes de flèche, deux lames ou lamelles tronquées, quelques herminettes trapézoïdales et têtes de hache taillées complètent l'inventaire. L'industrie en calcaire comprend des broyeurs, une extrémité de hache ou de herminette polie et plusieurs galets travaillés, des houes, quelques polissoirs et deux galets peints à l'ocre rouge. Le travail de l'os comprend des percuteurs tendres, plusieurs poinçons ou pointes, des sagaies à base arrondie aménagée, des petits tubes, deux pièces limées, un perçoir ou encore une spatule. La coquille d'œuf d'autruche* est très peu représentée avec quelques fragments, dont certains décorés de points ou de hachures, et quelques perles. De rares coquilles marines travaillées ont été identifiées. La céramique n'est pas très abondante. Le dégraissant de la pâte contient de la coquille pilée et du calcaire. La cuisson diffère d'un récipient à l'autre. L'épaisseur des tessons est comprise entre 4,4 et 13,2 $\mathrm{mm}$. Des tessons montrent des traces de finition par polissage, brunissage et, dans certains cas, par ajout d'un enduit. Le décor, très rare, est fait par impressions (rangées de points). Les tessons indiquent des récipients de taille variable avec un diamètre allant de 10 à $30 \mathrm{~cm}$; les vaisselles sont de forme hémisphérique, assez grandes, ouvertes, et probablement à fond arrondi. Il n'y avait aucune indication de col ou d'éléments de préhension mais quelques tessons présentaient un ourlet arrondi ou aplati.

5 La faune a été étudiée par E.S. Higgs (1967). La présence de la chèvre domestique est attestée par un fragment de cheville osseuse, tandis que certains ossements pourraient appartenir au mouton. Etant donné que les os de chèvres, de moutons et de mouflons à manchettes ont été regroupés sous le nom de Caprinés, nous n’avons aucune idée de 
leurs quantités respectives (1 723 restes osseux au Néolithique). À côté de cette faune domestique on trouve le chacal, l'antilope bubale, des rongeurs, quelques gazelles, lagomorphes et chats sauvages. Les bovins sauvages (Bos primigenius) sont moins fréquents que dans les niveaux précédents, et aucun reste osseux ne semble se référer à des bœufs domestiques. La collecte de mollusques marins est représentée par la très fréquente présence de coquilles de Patella et de Trochus.

Les restes fauniques d'Haua Fteah ont été réexaminés en 1985 (Klein \& Scott 1986). Les auteurs sont certains d'avoir bien identifié les ossements de chèvre domestique, mais un doute subsiste quant aux autres restes osseux qui pourraient, selon eux, tout aussi bien appartenir à des moutons domestiques qu'à de petits mouflons à manchettes. Ces restes problématiques ont été trouvés dans les niveaux néolithiques mais également dans les niveaux libyco-capsiens (4 restes) et, plus étonnant, dans les couches ibéromaurusiennes (12 restes). En ce qui concerne un os de chèvre domestique trouvé dans les niveaux épipaléolithiques libyco-capsiens en plus des niveaux néolithiques, les auteurs pensent qu'il provient des couches supérieures de la stratigraphie. R. Klein et K. Scott estiment que les méthodes de fouille ont pu provoquer de telles intrusions et préfèrent rester prudents sur le début de la domestication animale à Haua Fteah.

7 En 2007, l'Université de Cambridge a lancé le Cyrenaican Prehistory Project (CPP) qui a pour objectif de réétudier la stratigraphie d'Haua Fteah, grâce à une nouvelle fouille, et de reconstruire les paléoenvironnements de ces 200000 dernières années autour de la grotte. Des macrorestes botaniques ont été prélevés des couches holocènes de la nouvelle fouille puis analysés. Les principaux restes végétaux dans les contextes libycocapsiens sont représentés par des graines du Pin d'Alep (Pinus halepensis), suivis par des légumineuses sauvages (Vicia sp., Lathyrus sp., Lens sp.) puis par des arbres et arbustes méditerranéens (Juniperus phoenicea, Pistacia lentiscus, Myrtus communis) (Barker \& al. $2009 ;$ 2010). Les couches néolithiques n'ont livré aucun macroreste de céréale domestique contrairement aux couches épipaléolithiques du Libyco-capsien; malheureusement les datations C14 des quelques graines d'orge trouvées en 2007 démontrent que ce sont des intrusions très récentes (Barker \& al. 2010, p.78). D'autres restes d'orge (Hordeum vulgare) et de blé (Triticum durum) domestiqués, également trouvés dans les contextes capsiens doivent être soumis à datation directe (Barker \& al. 2010). Les nouvelles datations effectuées sur charbons par le CPP pour les couches néolithiques d'Haua Fteah indiquent les $7^{\mathrm{e}}$ et $6^{\mathrm{e}}$ millénaires $\mathrm{BP}$, c'est à dire les $6 \mathrm{e}$ et $5^{\mathrm{e}}$ millénaires avant notre ère (Barker \& al. 2009) : $6917 \pm 31 \mathrm{BP}, 6505 \pm 33 \mathrm{BP}, 6413 \pm 32$ BP et $6115 \pm 31$ BP pour la couche VIII ; $5759 \pm 28$ BP, $5521 \pm 32$ BP et $5462 \pm 30$ BP pour la couche VI (fig. 1).

\section{Abou Tamsa}

En 2006, la Mission Archéologique Française en Libye a été la première à relancer les recherches préhistoriques en Cyrénaïque avec l'étude du site d'Abou Tamsa (de Faucamberge 2010), situé à quelques centaines de mètres d'Haua Fteah. Un sondage sous l'abri d'Abou Tamsa a été réalisé en 2006 et 2007. La stratigraphie de $120 \mathrm{~cm}$ de profondeur a révélé quatre niveaux sédimentaires : le niveau I est le niveau de surface représenté par une terre sableuse de couleur beige clair qui s'est à certains endroits infiltrée sur plusieurs centimètres de profondeur; le niveau II est une terre sablonneuse très fine à pulvérulente, interrompue aux alentours de $40 \mathrm{~cm}$ par le niveau 
III, un «niveau coquillier ", très dur, résultant de la cémentation des nombreux débris de coquilles de gastéropodes avec le sédiment. Ce niveau se termine aux alentours de $50 \mathrm{~cm}$ de profondeur pour laisser place à un autre niveau sédimentaire IV, de texture sablonneuse identique au niveau II. La fouille s'est arrêtée à $120 \mathrm{~cm}$ de profondeur sans atteindre le lit rocheux.

9 La présence de restes d'ovicaprinés domestiques et l'étude du matériel archéologique permettent de rattacher la totalité du sondage à la période néolithique. Trois datations absolues ont été obtenues sur coquilles de patelle : $7695 \pm 60$ BP et $7275 \pm 40 \mathrm{BP}$ pour la couche IV et $6605 \pm 40$ BP pour la couche III (fig. 1).

L'assemblage se compose d'un éventail d'éléments caractéristiques : industrie lithique, industrie en pierre polie, industrie osseuse et industrie céramique. L'industrie en pierre taillée compte environ 1200 pièces Ethiques, dont près de la moitié se rapporte au débitage. Le reste de l'industrie comprend toutes les grandes classes d'outils : éclats, lames et lamelles retouchés, pointes, burins, grattoirs, perçoirs, racloirs, géométriques, micro-burins; cette industrie est cependant nettement dominée par les éclats retouchés, ainsi que par les coches et denticulés. L'industrie en pierre polie comprend une trentaine d'éléments en calcaire : quelques meules (entières et fragmentaires), une vingtaine de broyeurs et molettes ainsi que de petits galets utilisés comme polissoirs. L'industrie en os comprend une quarantaine d'éléments, représentés, entre autres, par des pointes, des poinçons, quelques lissoirs, perçoirs et une unique spatule. Le sondage a également livré quelques coquilles de gastéropodes marins et terrestres percées, et une rondelle en coquille d'œuf d'autruche. Environ trente tessons de céramique ont été mis au jour : trois reconstructions de la forme originelle du récipient indiquent des vaisselles de taille modeste, de forme hémisphérique et très certainement ouvertes. La grande majorité des poteries semble avoir été dégraissée avec des coquilles de gastéropodes broyées. La cuisson est variable, et certaines poteries semblent avoir bénéficié d'un traitement particulier de leur surface externe : décor, engobe, polissage.

\begin{tabular}{|l|l|l|l|l|l|l|}
\hline Site & Niveaux & Auteurs & Echantillon & Matériau & $\begin{array}{l}\text { Datation } \\
\text { BP }\end{array}$ & $\begin{array}{l}\text { Date cal BC } \\
(2 \sigma)\end{array}$ \\
\hline $\begin{array}{l}\text { Flaua } \\
\text { Fteah }\end{array}$ & VI (sommet) & McBurney 1967 & NPL 41 & Charbon & $4860 \pm 97$ & $3938-3375$ \\
\hline Haua Fteah & VI & Barker \& al. 2009 & OxA-18676 & $\begin{array}{l}\text { Zygophyllum } \\
\text { sp. }\end{array}$ & $5462 \pm 30$ & $4356-4259$ \\
\hline Haua Fteah & VI & Barker \& al. 2009 & OxA-18794 & $\begin{array}{l}\text { Zygophyllum } \\
\text { sp. }\end{array}$ & $5521 \pm 32$ & $4449-4332$ \\
\hline Haua Fteah & VI & Barker \& al. 2009 & OxA-18675 & $\begin{array}{l}\text { Zygophyllum } \\
\text { sp. }\end{array}$ & $5759 \pm 28$ & $4692-4534$ \\
\hline Haua Fteah & $\begin{array}{l}\text { VI } \text { (moitié } \\
\text { inférieure) }\end{array}$ & McBurney 1967 & NPL 40 & Charbon & 5800 & 498 \\
\hline Haua Fteah & VIII & Barker \& al. 2009 & OxA-18667 & Suaeda sp. & $6115 \pm 31$ & $5207-4948$ \\
\hline
\end{tabular}




\begin{tabular}{|l|l|l|l|l|l|l|}
\hline Haua Fteah & $\begin{array}{l}\text { VIII (moitié } \\
\text { supérieure) }\end{array}$ & McBurney 1967 & NPL 42 & Charbon & $\begin{array}{l}6370 \quad \pm \\
103\end{array}$ & $5528-5067$ \\
\hline Haua Fteah & VIII & Barker \& al. 2009 & OxA-19028 & $\begin{array}{l}\text { Rhus cf. } \\
\text { tripartita }\end{array}$ & $6413 \pm 32$ & $5470-5327$ \\
\hline Haua Fteah & VIII & Barker \& al. 2009 & OxA-18674 & Ephedra sp. & $6505 \pm 33$ & $5532-5376$ \\
\hline $\begin{array}{l}\text { Abou } \\
\text { Tamsa }\end{array}$ & III (45 cm) & $\begin{array}{l}\text { De Faucamberge } \\
2010\end{array}$ & Pa2468 & Patella & $6605 \pm 40$ & $5314-4874$ \\
\hline Haua Fteah & $\begin{array}{l}\text { VIII (moitié } \\
\text { inférieure) }\end{array}$ & McBurney 1967 & W 98 & Charbon & $6800 \quad \pm$ & $6430-5033$ \\
\hline Haua Fteah & VIII & Barker \& al. 2009 & OxA-18673 & $\begin{array}{l}\text { Rhus } \\
\text { tripartita }\end{array}$ & $6917 \pm 31$ & $5877-5729$ \\
\hline $\begin{array}{l}\text { Abou } \\
\text { Tamsa }\end{array}$ & IV (90 cm) & $\begin{array}{l}\text { Dc Faucamberge } \\
2010\end{array}$ & Pa2467 & Patella & $7275 \pm 40$ & $5931-5562$ \\
\hline $\begin{array}{l}\text { Abou } \\
\text { Tamsa }\end{array}$ & IV (105 cm) & $\begin{array}{l}\text { De Faucamberge } \\
2010\end{array}$ & Pa2472 & Patella & $7695 \pm 60$ & $6380-5960$ \\
\hline
\end{tabular}

Figure 1 : TABLEAU des dATATIONS ABSOLUES DES COUCHES HOLOCÈNES D'HAUA FTEAH DONNÉES EN ÂgE BP STANDARD (MCBURNEY 1967) ET CALIBRÉES GRÂCE AU LOgICIEL OXCAL V4.1.4 (INTCAL 09) À DEUX SIgMAS ; DATATIONS OBTENUES PAR LE CPP (BARKER \& AL. 2009) SUR CHARBONS ET LEURS CALIBRATIONS ; DATATIONS D'ABOU TAMSA, LES CALIBRATIONS ONT ÉTÉ RÉALISÉES PAR J.-F. SALIÈgE (DELTA R $58 \pm 85$ ) (DE FAUCAMBerge 2010).

11 Les restes fauniques recueillis dans le sondage représentent un demi-millier de pièces osseuses entières et fragmentaires ; mais seuls seize éléments (articulations osseuses et dents) ont pu être identifiés (fig. 2). Les chèvres (Capra hircus) et/ou moutons (Ovis aries) sont représentés par trois restes. Deux phalanges et un fragment de molaire se réfèrent soit à des ovicaprinés domestiques soit au mouflon à manchettes ; les autres ossements se rapportent à des bovidés sauvages et à des lagomorphes. Les gastéropodes terrestres (Helix, Rumina) et marins (Patella, Trochus) sont en réalité les espèces quantitativement les plus représentées.

\begin{tabular}{|c|c|c|c|c|c|c|c|c|}
\hline & $\begin{array}{l}\text { Chèvre } \\
\text { ou } \\
\text { Mouton }\end{array}$ & $\begin{array}{l}\text { Mouflon } \\
\text { à } \\
\text { manchette }\end{array}$ & $\begin{array}{l}\text { Ruminant } \\
\text { de petite } \\
\text { staille }\end{array}$ & $\begin{array}{l}\text { Ruminant } \\
\text { (Antilope } \\
\text { bubale?) }\end{array}$ & Artiodactyle & Lagomorphe & Lézard? & Crabe \\
\hline Calcanéum droit & 1 & & & & & & & \\
\hline $\begin{array}{l}\text { Molaire inférieure } \\
(M / 1 ?) \text { avec pli } \\
\text { caprin }\end{array}$ & 1 & & & & & & & \\
\hline $\begin{array}{l}\text { Fragment distal d'os } \\
\text { canon juvénile }\end{array}$ & 1 & & & 1 & & & & \\
\hline
\end{tabular}




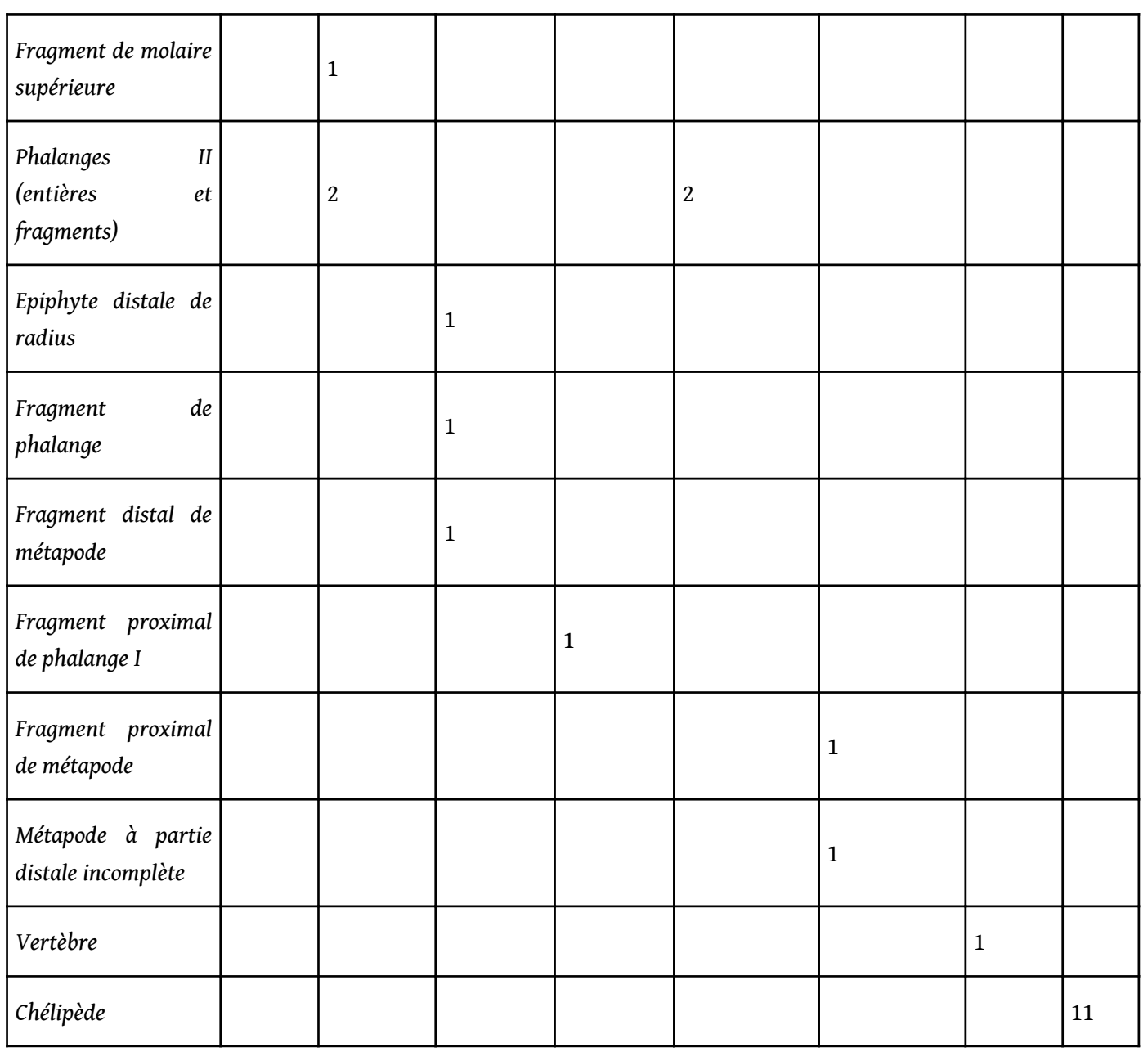

Figure 2 : RESTES FAUNIQUES dU SONDAgE d'ABOU TAMSA IDENTIfIÉS PAR C. GUÉRIN (DE FAUCAMBERgE 2010).

\section{Conclusion}

12 Les premières recherches effectuées sur le site d'Abou Tamsa permettent de confirmer et d'affiner les données obtenues à Haua Fteah concernant le début du Néolithique. En effet, la calibration de la date de $6800 \pm 350$ BP obtenue par McBurney donnait un intervalle très large de 1400 ans [6 430-5033 cal BC]. La datation de $7275 \pm 40 \mathrm{BP}$ obtenue à Abou Tamsa donne un intervalle calibré plus précis pour le début de la domestication animale [5 931-5 $562 \mathrm{cal} \mathrm{BC]}$; il en va de même des nouvelles datations effectuées par le CPP sur la couche VIII, qui indiquent des dates comprises entre 5877 et $4948 \mathrm{cal} \mathrm{BC}$ (fig. 1).

On peut ainsi en déduire que le Néolithique, caractérisé par l'élevage de petit bétail, commence dans la première moitié du $6^{\mathrm{e}}$ millénaire cal $\mathrm{BC}$, se plaçant ainsi parmi les plus anciennes évidences de domestication animale en Afrique du nord-est. Il reste encore à déterminer une éventuelle domestication du bœuf dans cette région ainsi que le début de la domestication végétale, sachant qu'une ancienne céréaliculture semble tout à fait plausible ici, la Cyrénaïque étant l'un des habitats naturels de l'orge sauvage. 
'autre part, si l'on s'appuie sur les données provenant d'Abou Tamsa, la céramique, recueillie dans la couche IV au niveau daté de $7695 \pm 60 \mathrm{BP}$, aurait été produite dans la seconde moitié du $7^{\mathrm{e}}$ millénaire cal BC [6380 - $5960 \mathrm{cal} \mathrm{BC}$ ], affinant là encore les résultats obtenus par McBurney. Les fouilles et analyses en cours à Haua Fteah et à Abou Tamsa apporteront de nouvelles informations concernant la néolithisation de la Cyrénaïque, qui, d'après les évidences archéologiques, a commencé très tôt dans cette partie littorale et nord-orientale de l'Afrique.

\section{BIBLIOGRAPHIE}

BARKER G., ANTONIADOU A., ARMITAGE S., BROOKS I., CANDY I., CONNELL K., DOUKA K., DRAKE N., FARR L., HILL E., HUNT C., INGLIS R., JONES S., LANE C., LUCARINI G., MENEELY J., MORALES J., MUTRI G., PRENDERGAST A., RABETT R., READE H., REYNOLDS T., RUSSELL N., SIMPSON D., SMITH B., STIMPSON C., TWATI M. \& WHITE K., 2010 - "The Cyrenaican Prehistory Project 2010 : the fourth season of investigations of the Haua Fteah cave and its landscape, and further results from the 2007-2009 fieldwork", Libyan Studies, volume 41, p. 63-88.

BARKER G., ANTONIADOU A., BARTON H., BROOKS I., CANDY I., DRAKE N., FARR L., HUNT C., ABDULHAMID IBRAHIM A., INGLIS R., JONES S., MORALES J., MORLEY I., MUTRI G., RABETT R., REYNOLDS T., SIMPSON D., TWATI M. \& WHITE K., 2009 - "The Cyrenaican Prehistory Project 2009 : the third season of investigations of the Haua Fteah cave and its landscape, and further results from the 2007-2008 fieldwork", Libyan Studies, volume 40, p. 55-94.

DE FAUCAMBERGE E., 2010 - Abou Tamsa : étude d'un nouveau site néolithique en Cyrénaïque (Libye). Thèse de Doctorat, Université de Paris I Panthéon-Sorbonne, 351 p.

HIGGS E. S. 1967 - "Environment and chronology - the evidence from mammalian fauna". In : C. B. M. McBurney (Ed), The Haua Fteah (Cyrenaica) and the Stone Age of the south-east Mediterranean. Cambridge University Press (London), p. 16-44.

KLEIN R. G. \& SCOTT K., 1986 - "Re-analysis of faunal assemblages from the Haua Fteah and other late Quaternary archaeological sites in Cyrenaican Libya", Journal of Archaeological Science, vol. 13, p. 515-542.

KLEINSMIEDE W. F. J. \& VAN DEN BERG N. J., 1968 - "Surface geology of the Jabal al Akhdar, Northern Cyrenaica, Libya", in : F.T. Barr (Ed), Geology and Archaeology of northern Cyrenaica, Libya. Petroleum Exploration Society of Libya, p. 115-123.

MC BYRNEY C. B. M., 1967 - The Haua Fteah (Cyrenaica) and the Stone Age of the south-east Mediterranean, Cambridge University Press (London), 387 p.

\section{INDEX}

Mots-clés : Libye, Néolithique 